\title{
Interfacing transition metal dichalcogenides with carbon nanodots for managing photoinduced energy and charge-transfer processes
}

\author{
Lorenzo Vallan, ${ }^{\ddagger \#}$ Ruben Canton-Vitoria, ${ }^{\S \#}$ Habtom B. Gobeze, ${ }^{\text {ॠ\# }}$ Youngwoo Jang, Raul Arenal, ${ }^{*}$, $¥$ \\ Ana M. Benito, ${ }^{\ddagger}$ Wolfgang K. Maser, ${ }^{* \ddagger}$ Francis D’ Souza, ${ }^{* \star}$ Nikos Tagmatarchis*§ \\ ${ }^{\S}$ Theoretical and Physical Chemistry Institute, National Hellenic Research Foundation, 11635 Athens, Greece. \\ ${ }^{\ddagger}$ Instituto de Carboquímica, Consejo Superior de Investigaciones Científicas, 50018 Zaragoza, Spain. \\ " Department of Chemistry, University of North Texas, 305070 Denton, TX 76203-5017, USA. \\ ${ }^{\$}$ Laboratorio di Microscopias Avanzadas, Instituto de Nanociencia de Aragon, Universidad de Zaragoza, 50018 Zaragoza, \\ Spain. \\ ¥ ARAID Foundation, 50018, Zaragoza, Spain.
}

\begin{abstract}
Exfoliated semiconducting $\mathrm{MoS}_{2}$ and $\mathrm{WS}_{2}$ were covalently functionalized with 1,2-dithiolane-modified carbon nanodots (CNDs). The newly synthesized CND-MoS ${ }_{2}$ and $\mathrm{CND}^{-\mathrm{WS}_{2}}$ hybrids were characterized by spectroscopic, thermal and electron microscopy imaging methods. Based on electronic absorption and fluorescence emission spectroscopy, modulation of the optoelectronic properties of TMDs by interfacing with CNDs was accomplished. Electrochemical studies revealed facile oxidation of $\mathrm{MoS}_{2}$ over $\mathrm{WS}_{2}$ in the examined hybrids, suggesting it to be better electron donor. Excited state events, investigated by femtosecond transient absorption spectroscopic studies, revealed ultrafast energy transfer from photoexcited CNDs to both $\mathrm{MoS}_{2}$ and $\mathrm{WS}_{2}$. Interest-

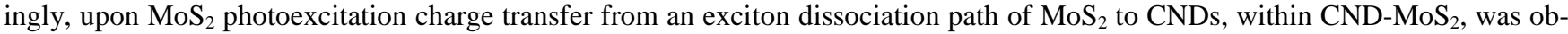
served. However, such process in CND-WS ${ }_{2}$ was found to be absent due to energetic reasons. The present study highlights the importance of TMD-derived donor-acceptor hybrids in light energy harvesting and optoelectronic applications. Furthermore, the fundamental information obtained from the current results will benefit design strategies and impact the development of additional TMDbased hybrid materials to efficiently manage and perform in electron-transfer processes.
\end{abstract}

\section{INTRODUCTION}

Carbon nanodots (CNDs) are recognized as promising materials for energy conversion applications. ${ }^{1-3}$ In general, CNDs are photochemically stable, ${ }^{4}$ show varied solubilty in aqueous and organic media ${ }^{5}$ and possess size in the order of $1-10 \mathrm{~nm} .^{6,7}$ The CNDs are abundantly prepared by economic, facile and environmental benign routes, mainly involving polycondensation reactions of small organic molecules at medium or elevated temperatures under hydrothermal or microwave irradiation conditions. ${ }^{8-10}$ Importantly, the surface of CNDs can be decorated with diverse functional units, which can serve as anchors to conjugate other species en route the preparation of functional hybrid nanomaterials. Notably, depending on the precursors employed for the synthesis, the periphery of the surface of CNDs can be decorated with diverse functional units, which can serve as anchors to conjugate other species en route the preparation of functional hybrid nanomaterials. For example, CNDs have been covalently conjugated with carbon nanotubes,$^{11}$ porphyrins $^{12}$ and extended tetrathiafulvalene (exTTF) ${ }^{13}$ and found to participate in photoinduced charge-transfer processes. Among the marked characteristics of CNDs are the broad and tunable absorption in the visible region and the intrinsic photoluminescence, ${ }^{14}$ while interestingly, CNDs can act as either electron donors or acceptors, owed to their bivalent redox character..$^{15}$ This is to say that upon photoirradiation, CNDs donate electrons when assembled with carbon nanotubes or perylenediimides, ${ }^{11}$, 16,17 and accept electrons upon interaction with porphyrins and exTTF. ${ }^{12,13,18}$

Conversely, the fascination of transition metal dichalcogenides (TMDs) for energy-related applications, ${ }^{19-22}$ attributed to their astounding optoelectronic properties and high electrical conductivity $^{23}$ has become progressively apparent. Molybdenum disulfide $\mathrm{MoS}_{2}$ and tungsten disulfide $\mathrm{WS}_{2}$, as the most fascinated and examined TMDs, consist of an atomic thick layer of transition metals sandwiched by two atomic layers of sulfur atoms. ${ }^{24,}{ }^{25}$ Even though the crystal structure of TMDs can greatly vary, depending on the number of d-electrons of the transition metal, the most commonly observed polytypes are those with trigonal prismatic $D_{3 h}$ symmetry possessing semiconducting properties and with octahedral $O_{h}$ coordination which show metallic behavior. ${ }^{26}$ Delaminated TMDs can be obtained by exfoliation of the bulk counterpart and depending on the exfoliating agent employed, different polytypes are derived. For instance, treatment of bulk TMDs with organometallic media results to the metastable metallic polytype which converts to the semiconducting one by annealing, with electron-transfer phenomena governing the exfoliation process. ${ }^{27-31}$ On the other hand, chlorosulfonic acid acting as Bronstead acid partially protonates the sulfur atoms of $\mathrm{MoS}_{2}$ and $\mathrm{WS}_{2}$, without oxidizing them, yielding semiconducting exfoliated nanosheets due to the 
development of repulsive electrostatic forces between the layers of TMDs. ${ }^{32}$

Managing and controlling the electronic properties of semiconducting TMDs, which are governed by excitonic transitions, is mandatory for their realization as prototype devices in energy-related applciations. To this end, among other sophisticated and complicated processes employed in nanosized semiconducting materials, a facile yet straightforward approach to gain precise control over the optoelectronic properties is by decorating the surface of TMDs with photo- and/or electro-active species and tuning the charge-carrier density. ${ }^{33}$ Hence, covalent functionalization unlocks the potentiality of TMDs by opening new opportunities and broadening the spectrum of applications. ${ }^{34-39}$ In a prominent example, exploiting the high binding affinity of 1,2-dithiolanes for transition metal atoms with chalcogen vacant sites, the covalent functionalization of exfoliated semiconducting TMDs was accomplished. ${ }^{39}$ Nevertheless, interfacing TMDs with photoactive species via robust covalent bonding toward the development of advanced hybrid materials has yet to be fully advanced. Veritably, the development of such hybrids is timely and surely deserves investigation, especially in the context of their ability to function as donor-acceptor systems upon photoillumination.

With all the above in mind, CNDs and TMDs as two highly promising nanomaterials for energy conversion, each one possessing unique characteristics and properties, were combined via robust covalent bonding en route the realization of novel donor-acceptor hybrids. Specifically, herein we report on the conjugation of modified CNDs incorporating 1,2-dithiolane moieties at the edges of exfoliated semiconducting $\mathrm{MoS}_{2}$ and $\mathrm{WS}_{2}$ and perform a comprehensive photophysical study. With the current approach, modulation and engineering of the optoelectronic properties of photoexcited TMDs was accomplished, by employing CNDs as electron acceptors, via photoinduced electron transfer processes to occur from $\mathrm{MoS}_{2}$, but not from $\mathrm{WS}_{2}$ to CNDs. The newly prepared CND-MoS 2 and CND-WS hybrid materials were fully characterized by complementary spectroscopic, thermal and electron microscopy imaging means, undoubtfully verifying the structures derived by the functionalization process. The optical and redox properties of CND- $\mathrm{MoS}_{2}$ and CND-WS $\mathrm{W}_{2}$ were probed by electronic absorption, fluorescence emission spectroscopy and electrochemistry, respectively, revealing the existence of excited state intrahybrid electronic interactions between the two species, while also showing that $\mathrm{MoS}_{2}$ was a better electron donor compared to $\mathrm{WS}_{2}$. Furthermore, with the aid of femtosecond transient spectroscopy by exciting the TMD part within the hybrid materials, strong electronic interactions between the two species leading to photoinduced charge-separation only within $\mathrm{CND}-\mathrm{MoS}_{2}$ but not for CND-WS $\mathrm{W}_{2}$ were identified. In addition, upon excitation of CNDs in CND-TMD ultrafast energy transfer from excited CNDs to both $\mathrm{MoS}_{2}$ and $\mathrm{WS}_{2}$ was identified. The fundamental information obtained from the current study will impact the development of additional TMD-based hybrid materials to efficiently manage and perform in electron-transfer processes aiming to photovoltaic and solar energy related applications.

\section{RESULTS AND DISCUSSION}

Initially, CNDs featuring plethora of free amine groups were prepared by polycondensation of citric acid and ethylenediamine, followed by addition of butylamine. The latter enhances the lipophilicity of CNDs, by introducing butyl units, while at the same time consumes all remaining carboxylic acid groups, stopping the growth of the nanoparticles. Next, lipoic acid activated by (3-dimethylaminopropyl)-N'-ethylcarbodiimide was condensed to yield modified CNDs bearing 1,2-dithiolanes as substituents on the periphery of their structure. Spectroscopic characterization by ${ }^{1} \mathrm{H}$ NMR and IR (Supporting Information, Figures S1, S2) verified the structure of modified CNDs and the success of synthesis. Further proof was delivered by the Kaiser test, where the amount of free amines on CNDs decreased significantly upon condensation with lipoic acid, from 1810 to 72 $\mu \mathrm{mol} / \mathrm{g}$. In parallel, bulk $\mathrm{MoS}_{2}$ and $\mathrm{WS}_{2}$ were wet exfoliated by chlorosulfonic acid, ${ }^{32}$ and following the functionalization methodology for TMDs with 1,2-dithiolanes, ${ }^{39-41}$ the conjugation of modified CNDs was accomplished, furnishing $\mathrm{CND}-\mathrm{MoS}_{2}$ and CND-WS ${ }_{2}$ according to Scheme 1.

Scheme 1. Illustrative preparation of CND-MoS 2 and CND$\mathrm{WS}_{2}$ upon covalent 1,2-dithiolane functionalization of exfoliated semiconducting $\mathrm{MoS}_{2}$ and $\mathrm{WS}_{2}$ nanosheets.

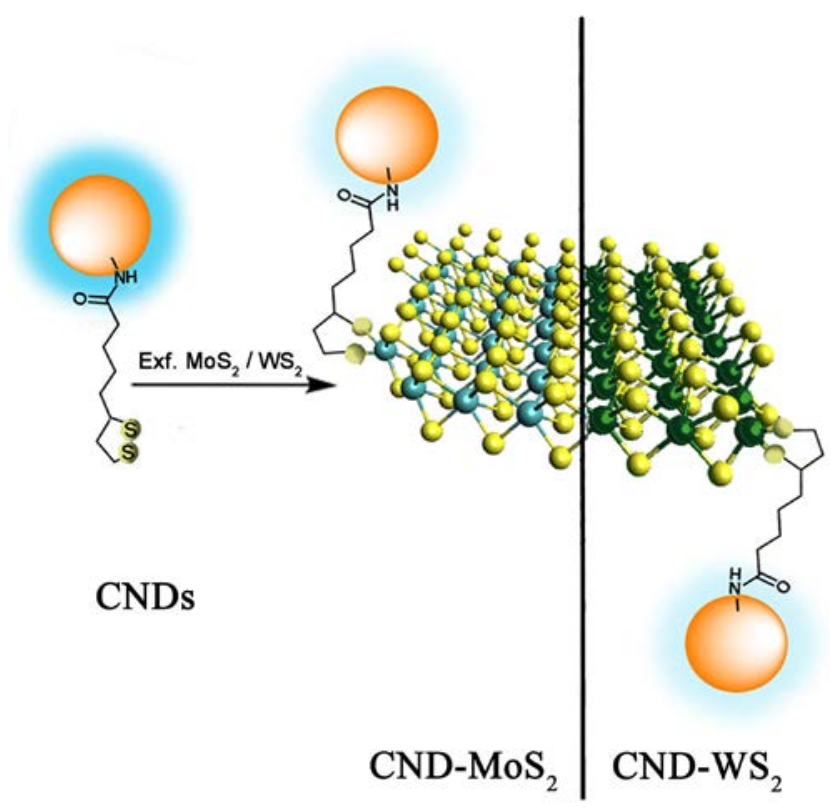

The newly derived hybrid materials found to be soluble in $\mathrm{xxx}(\mathrm{xxx} \mathrm{mg} / \mathrm{mL}), \mathrm{xxx}(\mathrm{xxx} \mathrm{mg} / \mathrm{mL})$ and $\mathrm{xxx}(\mathrm{xxx} \mathrm{mg} / \mathrm{mL})$, while remained insoluble in $\mathrm{xxx}$ and $\mathrm{xxx}$. Notably, the solubility of both CND-MoS $\mathrm{M}_{2}$ and CND-WS $\mathrm{W}_{2}$ in $\mathrm{xxx}$ remained unchanged for a period of several months, evidenced by the absence of any precipitation, hence proving their high stability.

Next, complementary characterization of CND-MoS 2 and CND-WS $\mathrm{S}_{2}$ by IR and Raman spectroscopy, thermogravimetric analysis (TGA) and transmission electron microscopy (TEM) imaging was performed. The presence of characteristic bands at 1640 and $1550 \mathrm{~cm}^{-1}$, related with stretching and bending modes of carbonyl amide vibrations, were evident in the ATR-IR spectrum of CND-MoS ${ }_{2}$ and CND-WS ${ }_{2}$ (Supporting Information, Figure S3). In addition, C-H stretching vibrations at 2960 and $2915 \mathrm{~cm}^{-1}$ due to the alkyl chain of 1,2-dithiolane were present. 

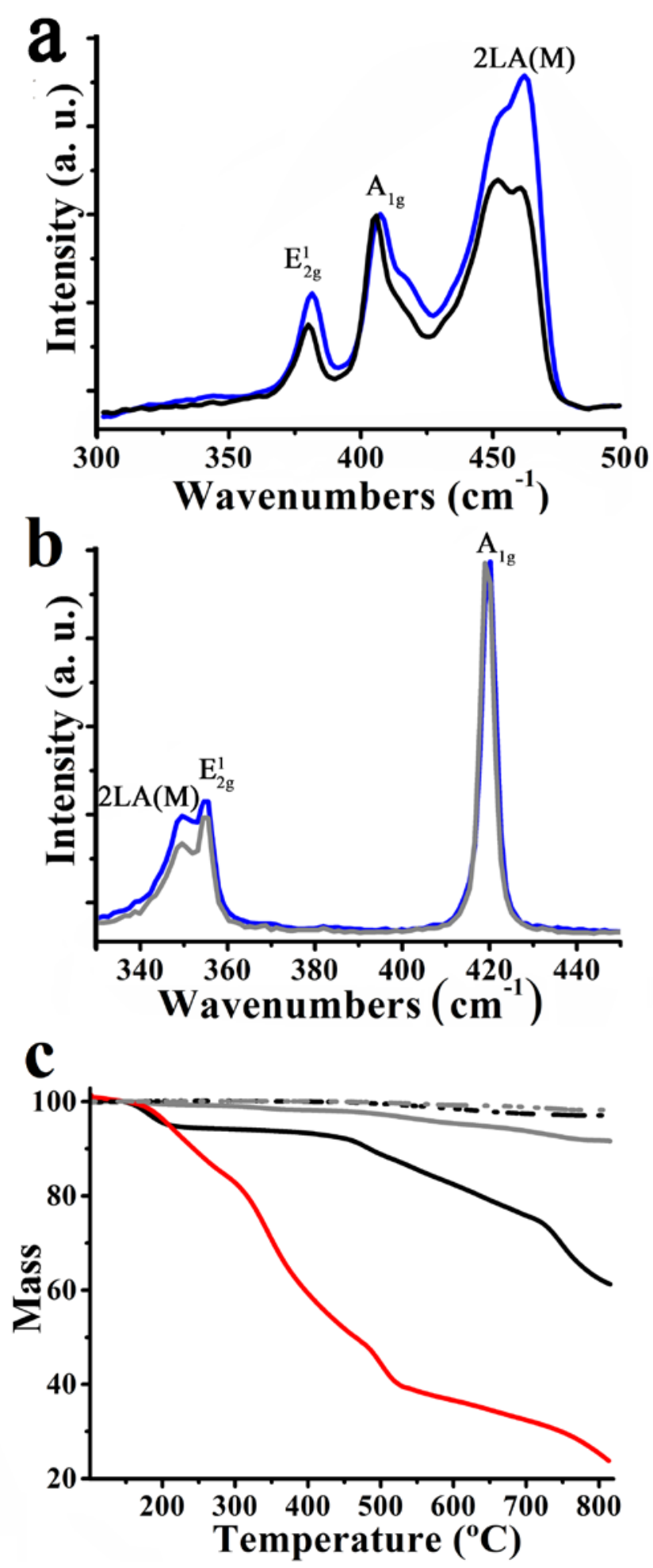

Figure 1. Raman spectra normalized at $\mathrm{A}^{1} \mathrm{~g}$ mode for (a) exfoliated $\mathrm{MoS}_{2}$ (blue) and CND-MoS 2 (black) at $\lambda_{\text {exc }} 633 \mathrm{~nm}$, and (b) exfoliated $\mathrm{WS}_{2}$ (blue) and CND-WS 2 (grey) at $\lambda_{\text {exc }} 514 \mathrm{~nm}$. (c) Thermographs for CNDs (red), exfoliated $\mathrm{MoS}_{2}$ (dotted black), exfoliated $\mathrm{WS}_{2}$ (dotted gray), CND-MoS 2 (black), and CND-WS 2 (grey).

Comparing Raman spectra of exfoliated $\mathrm{MoS}_{2}$ and CND$\mathrm{MoS}_{2}$, obtained upon excitation under on-resonance conditions at $633 \mathrm{~nm}$ and normalized at the $\mathrm{A}_{1 \mathrm{~g}}$ mode at $404 \mathrm{~cm}^{-1}$, it was found that the intensity of the 2LA(M) band associated to disorder and defects ${ }^{42}$ and located at $447 \mathrm{~cm}^{-1}$ was found decreased in $\mathrm{CND}-\mathrm{MoS}_{2}$ as compared to that owed to exfoliated $\mathrm{MoS}_{2}$ (Figure 1a). ${ }^{36,43}$ The latter comes as a result of the reduced number of S defects due to edge functionalization of $\mathrm{MoS}_{2} \cdot{ }^{39}$ Moreover, the absence of the characteristic phonon modes of metallic polytype $\mathrm{MoS}_{2}$ so-called $\mathrm{J}_{1}, \mathrm{~J}_{2}$ and $\mathrm{J}_{3}$ at 150,225 and $325 \mathrm{~cm}^{-1}$, respectively, ${ }^{44}, 45$ ascertained the semiconducting behavior of $\mathrm{MoS}_{2}$ in the CND-MoS 2 hybrid material. Regarding $\mathrm{CND} \mathrm{WS}_{2}$, bands due to $2 \mathrm{LA}(\mathrm{M}), \mathrm{E}^{1}{ }_{2 \mathrm{~g}}$, and $\mathrm{A}_{1 \mathrm{~g}}$, upon onresonance excitation at $514 \mathrm{~nm}$, were evident at 350, 354 and $419 \mathrm{~cm}^{-1}$, with the intensity of the 2LA(M) mode decreased by $20 \%$ as compared to exfoliated $\mathrm{WS}_{2}$ (Figure 1b). Furthermore, for both CND-MoS ${ }_{2}$ and CND-WS 2 , the $A_{1 g}$ and $E^{1}{ }_{2 g}$ modes found red-shifted by $1-2 \mathrm{~cm}^{-1}$ as compared to the values registered for exfoliated $\mathrm{MoS}_{2}$ and $\mathrm{WS}_{2}$ respectively. The latter is attributed to intrahybrid charge-transfer phenomena developed between the CNDs and the TMDs, in accordance with literature reports. ${ }^{46,}{ }^{47}$ Since CNDs are highly fluorescent, weak and broad Raman bands attributed to $-\mathrm{NC}=\mathrm{O}, \mathrm{C}=\mathrm{O}$ and $\mathrm{C}-\mathrm{H}$ units were observed for both CND-MoS ${ }_{2}$ and CND-WS only upon excitation at $1064 \mathrm{~nm}$ (Supporting Information, Figure S4).

The loading of CNDs conjugated onto $\mathrm{MoS}_{2}$ and $\mathrm{WS}_{2}$ in CND- $\mathrm{MoS}_{2}$ and CND-WS ${ }_{2}$ was evaluated by TGA (Figure 1c). The modified CNDs bearing 1,2-dithiolane units were thermally stable up to $200{ }^{\circ} \mathrm{C}$ under nitrogen atmosphere, while they lost $65 \%$ of mass at the temperature range $200-500{ }^{\circ} \mathrm{C}$. Since $\mathrm{MoS}_{2}$ and $\mathrm{WS}_{2}$ are thermally stable in that temperature range, the observed mass loss for CND- $\mathrm{MoS}_{2}$ and CND-WS $\mathrm{W}_{2}$, $7.5 \%$ and $3.0 \%$ respectively, is related to the decomposition of modified CNDs present in the two hybrids. Although this is a relatively small mass loss, it is consistent with the edge functionalization of the limited S vacant sites of $\mathrm{MoS}_{2}$ and $\mathrm{WS}_{2 .}{ }^{39}$

The CND- $\mathrm{MoS}_{2}$ and $\mathrm{CND}-\mathrm{WS}_{2}$ hybrids were morphologically imaged by HR-TEM. A few drops of a dispersion of the materials in hexane were deposited on the TEM grid and imaged after the solvent was evaporated. Extensive imaging of several different areas and flakes of the CND$\mathrm{MoS}_{2}$ and CND-WS $\mathrm{S}_{2}$ hybrid materials revealed that the size of $\mathrm{MoS}_{2}$ and $\mathrm{WS}_{2}$ is in the order of few hundred nanometres, e.g. around 200-400 nm (Supporting Information, Figure S4). Although mostly oligolayered flakes were observed, most likely due to restacking of the TMDs during the drying process of the sample after depositing it on the TEM grid, the presence of some monolayered ones were also identified. In order to get better insight on $\mathrm{CND}^{-\mathrm{MoS}_{2} \text { and CND-WS }}$, TEM studies complemented with spatially-resolved electron energy loss spectroscopy (EELS) were performed. Figures 2a and $2 \mathrm{f}$ show high angle annular dark field (HAADF) scanning TEM (STEM)

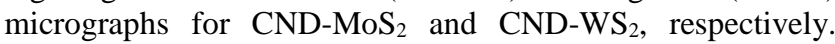
Based on the following spectroscopic/chemical TEM analyses, the bright small objects observed in these images was assigned to CNDs covalently anchored on TMD flakes. This is confirmed by energy dispersive X-ray spectroscopy (EDS) analyses (Figures 2b, g) and EELS (Figures 2c-e and 2h-j). Figure 2c displays an ADF micrograph of CND-MoS ${ }_{2}$ and an EELS spectrum-image (SPIM) was recorded in the red marked rectangular area. Three EEL spectra were extracted in the highlighted square regions (Figure 2e(i)-(iii)). Each of these three EEL spectra corresponds to the sum of 9 spectra (3x3 probe positions of the SPIM). The S- $\mathrm{L}_{2,3}$ and Mo-M edges are visible in the three spectra and correspond to $\mathrm{MoS}_{2} .^{48,49}$ It is worth mentioning that no $\mathrm{MoO}_{\mathrm{x}}$ was observed highlighting the 
high quality and purity of the materials. In addition, $\mathrm{C}$ was detected in specific areas, see the presence of the $\mathrm{C}-\mathrm{K}$ edge (Figure 2e(ii)-(iii)). This C-K edge, which is superposed to the Mo- $\mathrm{M}_{4,5}$ edge, is associated with the presence of CNDs within the CND-MoS 2 hybrid. The chemical C map obtained from the analysis of C-K edge (Figure $2 \mathrm{~d}$ ) clearly supports this finding. ${ }^{48,}$ ${ }^{49}$ Indeed, CNDs are observed not only in the ADF-STEM micrograph (Figure 2c) but also in this C-map (Figure 2d). Similar assays were performed for CND-WS $\mathrm{W}_{2}$ and from TEM analyses (Figures XXX Sup. Inf) the presence of CNDs attached on $\mathrm{WS}_{2}$ was confirmed.

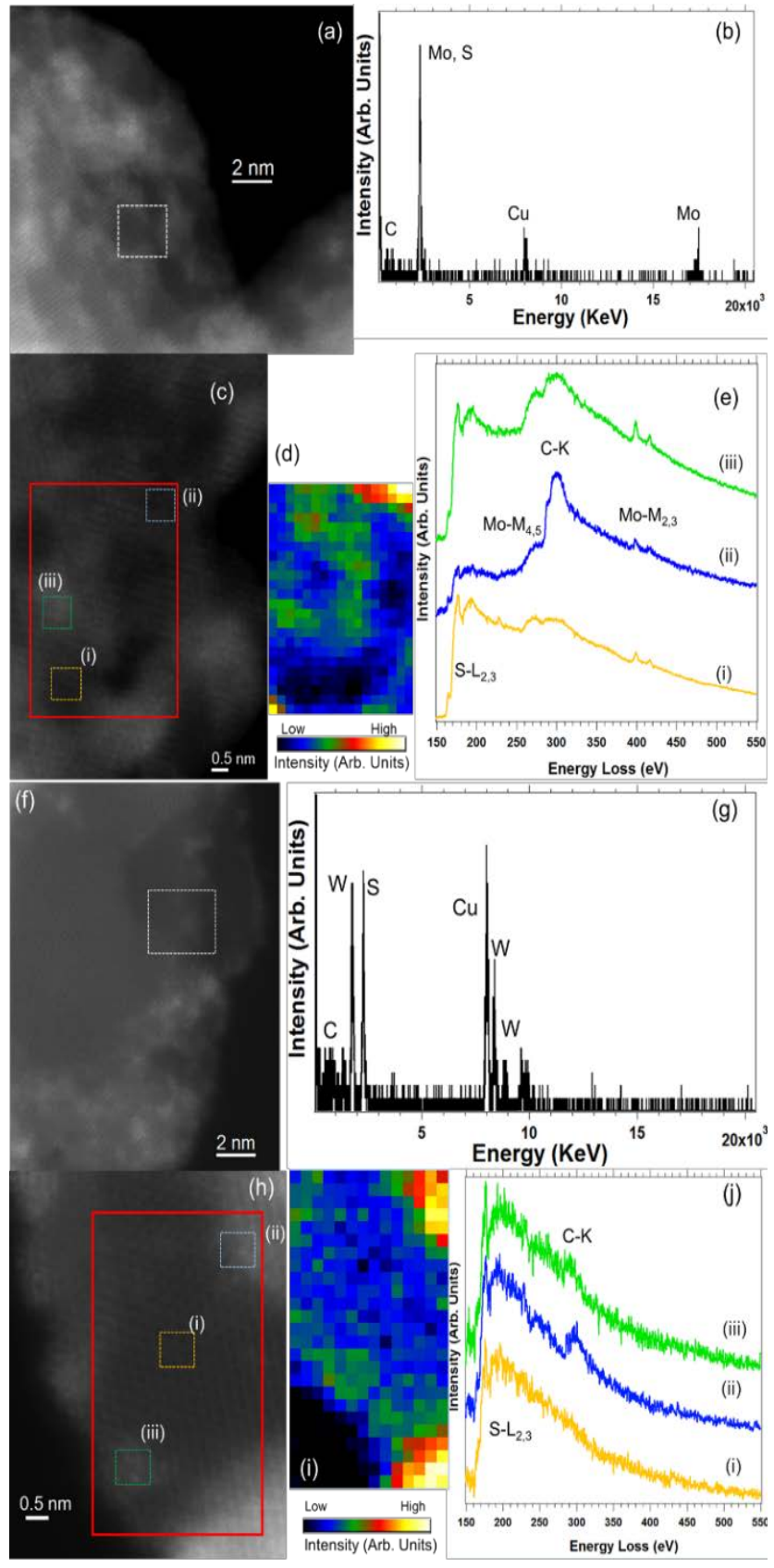

Figure 2. Representative HRSTEM-ADF images for (a, d) CND$\mathrm{MoS}_{2}$, and (f, h) CND-WS 2 . (b, g) EDS on the squared white areas in $(a, f)$, respectively. In the red regions of $(c, h)$ spectrum-images SR-EELS were recorded. (d, i) Carbon elemental mapsextracted from the integrated intensity of the C-K edge of the two EELS spectra-image recorded in the red areas in (c, h). (e) Three spectra from the sum of 9 (3x3) EELS extracted from the marked areas of the EELS SPIM of (c). The C-K edge ( $284 \mathrm{eV})$ is obs erved in (ii) and (iii) superposed with the Mo-M edge. The S-L2,3 and Mo-M edge of $\mathrm{MoS}_{2}$ are visible in the 3 spectra ((i)-(iii)). (j) Similar analysis for CND-WS 2 as in (e). Three spectra from the sum of 16 (4x4) EELS extracted from the EELS SPIM of (h), showing the S$\mathrm{L}_{2,3}$ and $\mathrm{C}-\mathrm{K}$ (in this case only in (ii) and (iii)) edges.
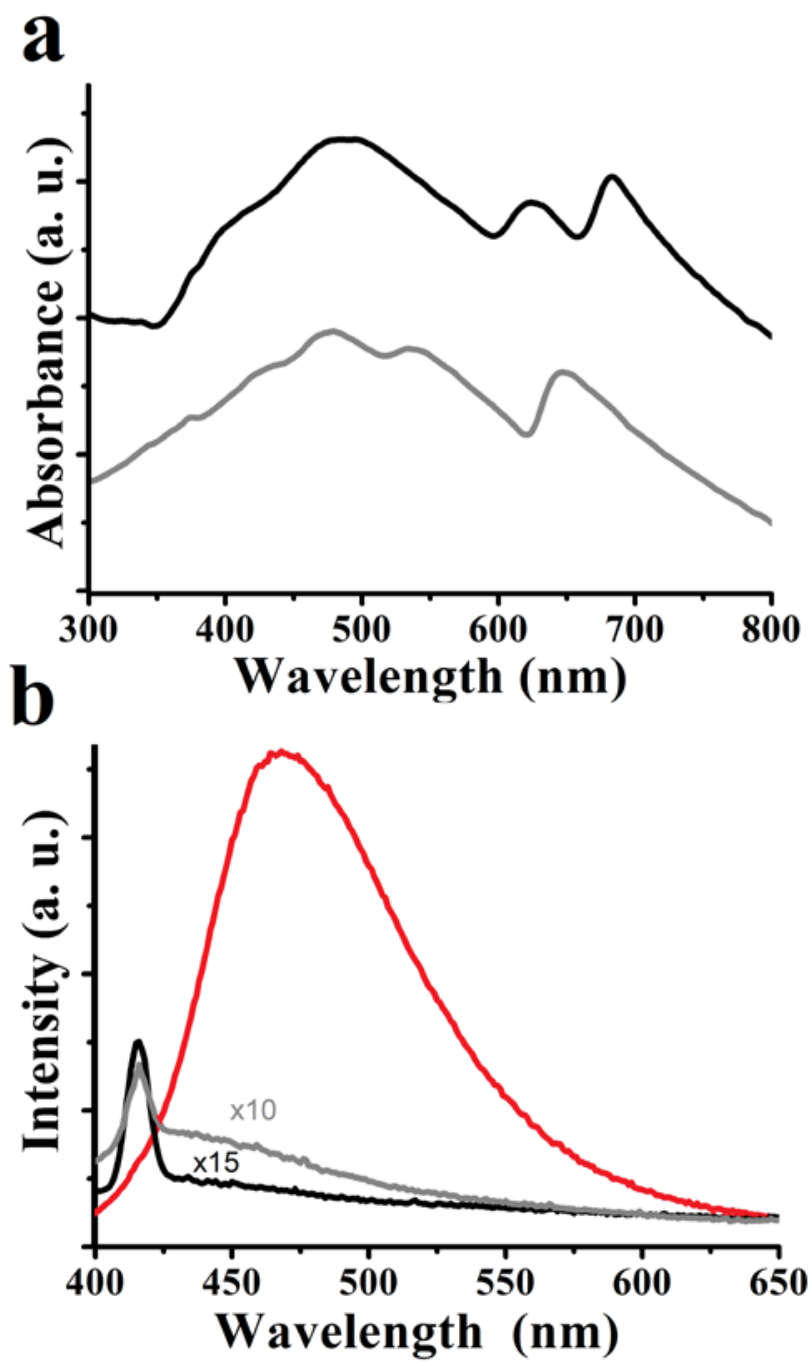

Figure 3. (a) Absorption and (b) emission spectra ( $\left.\lambda_{\text {exc }} 370 \mathrm{~nm}\right)$ of CND-MoS 2 (black), CND-WS 2 (grey) and modified CNDs (red), in DMF.

Focusing on the optical properties of CND-MoS 2 and CND$\mathrm{WS}_{2}$, electronic absorption and fluorescence assays were conducted. In more detail, in the UV-Vis spectra of CND-MoS and CND-WS ${ }_{2}$ characteristic bands centered at 680, 620, 485, $400 \mathrm{~nm}$, and 645, 535, 475, $420 \mathrm{~nm}$, respectively, corresponding to semiconducting $\mathrm{MoS}_{2}$ and $\mathrm{WS}_{2}$, in addition to continuous absorption throughout the visible region due to the presence of both TMDs and CNDs, were seen (Figure 3a). Unfortunately, the strong absorption features of TMDs masked the broad band of modified CNDs, appearing at $370 \mathrm{~nm}$ (Supporting Information, Figure S5), hence hampering to defer conclusive statements regarding possible intrahybrid electronic 
communication between the two species in the ground state. Nevertheless, the situation is rather clear in the excited state, where the strong emission of modified CNDs, centred at 470 $\mathrm{nm}$ upon excitation at $370 \mathrm{~nm}$, was found blue-shifted by 30 $\mathrm{nm}$, at $440 \mathrm{~nm}$, and quantitatively quenched in both CND-MoS and CND-WS 2 (Figure $3 b$ ). The later postulates the occurrence of an additional deactivation path for the singlet excited state of CNDs, through an energy or charge-transfer process. Next, analysis of the fluorescence emission decay profiles at $450 \mathrm{~nm}$ for the singlet excited state of CNDs upon excitation at $376 \mathrm{~nm}$, gave a monoexponentially fitted lifetime of 6.3 ns. However, the corresponding analysis for CND- $\mathrm{MoS}_{2}$ and CND-WS resulted in a biexponential decay, with the identification of major faster components with 1.2 and 1.1 ns lifetime, corresponding to the quenching of singlet excited state of CNDs in CND-MoS and CND-WS 2 , respectively.

The electrochemical behavior of CND- $\mathrm{MoS}_{2}$ and CND-WS was successively investigated in DMF (Supporting Information, Figure S6). The cyclic voltammogram (CV) of exfoliated $\mathrm{MoS}_{2}$ revealed irreversible oxidations at $E_{\mathrm{pa}}=-0.08$ and $0.48 \mathrm{~V}$ and reductions at $E_{\mathrm{pc}}=-1.20$ and $-1.69 \mathrm{~V} \mathrm{vs} \mathrm{Fc/Fc}$. In $\mathrm{CND}-\mathrm{MoS}_{2}$, the first oxidation wave was too broad to pick the peak potential, while the second one was better defined with an $E_{\mathrm{pa}}=0.54 \mathrm{~V}$. The two reductions were located at $E_{\mathrm{pc}}=-1.48$ and $-2.20 \mathrm{~V}$ as a consequence of the covalent functionalization. The CV of exfoliated $\mathrm{WS}_{2}$ revealed irreversible oxidations at $E_{\mathrm{pa}}=0.23$ and $0.44 \mathrm{~V}$ and reductions at $E_{\mathrm{pc}}=-1.40$ and $-1.98 \mathrm{~V}$. Upon covalent attachment of CNDs, the oxidation waves broadened, making it difficult to identify the peak potential, while the reduction was anodically shifted to $E_{\mathrm{pc}}=-1.37$ and $1.63 \mathrm{~V}$. Importantly, both $\mathrm{MoS}_{2}$ and $\mathrm{WS}_{2}$ were found to be electroactive and such property persisted upon chemical functionalization with CNDs. The facile oxidation of $\mathrm{MoS}_{2}$ over $\mathrm{WS}_{2}$ suggests it to be better electron donor. The CV of CNDs revealed no measurable electrochemical activity within the potential window. Differential spectral changes observed during the first oxidation and reduction of $\mathrm{MoS}_{2}$ and $\mathrm{WS}_{2}$ are shown at the Supporting Information, Figure S7. In both TMDs, reduction in peak intensity of the neutral species was observed, more so for $\mathrm{MoS}_{2}$ compared to $\mathrm{WS}_{2}$. Some positive spectral features in the $300-450 \mathrm{~nm}$ range were observed for $\mathrm{MoS}_{2}$ during oxidation.

The excited state events were probed by femtosecond pumpprobe transient absorption spectroscopy in DMF, where dispersion of the hybirds was appreciable. The samples were excited at $370 \mathrm{~nm}$ corresponding mainly to CNDs excitation and at $425 \mathrm{~nm}$ corresponding mainly to TMDs excitation. In agreement with literature reports, ${ }^{50-52}$ immediately after $425 \mathrm{~nm}$ excitation of exfoliated $\mathrm{MoS}_{2}$, three minima at 503, 637 and 696 $\mathrm{nm}$ due to excitonic transitions as seen in the absorption spectrum, and two maxima at 595 and $663 \mathrm{~nm}$ corresponding to induced absorption of $\mathrm{B}$ and $\mathrm{A}$ excitons, were observed (Supporting Information, Figure S8a). During the first 10 ps, all peaks experienced blue shift ascribed to cooling of hot excitons and/or interexcitonic interactions. In the case of exfoliated $\mathrm{WS}_{2}$, two minima at 545, and $652 \mathrm{~nm}$ (B and A excitons, by comparison with the absorption spectrum) and two maxima at 516 and $617 \mathrm{~nm}$ were observed (Supporting Information, Figure S8b). The peak positions also experienced a small blue-shift of $2 \mathrm{~nm}$ within the first $10 \mathrm{ps}$.

Figure 4 shows the transient absorption spectra and intensitywavelength maps of CND-MoS ${ }_{2}$ and $\mathrm{CND}-\mathrm{WS}_{2}$ dispersions in
DMF at $\lambda_{\text {exc }} 370 \mathrm{~nm}$ mainly exciting the CNDs. The transient spectra of CNDs ( $\lambda_{\text {exc }} 370 \mathrm{~nm}$ ) revealed positive peaks at 460 and $590 \mathrm{~nm}$ (Supporting Information, Figure S9a) originating from transitions involving excited CNDs. The decay of these peaks was rather slow consistent with the longer fluorescence lifetime of CNDs (6.3 ns). When exfoliated $\mathrm{MoS}_{2}$ and $\mathrm{WS}_{2}$ were excited at $370 \mathrm{~nm}$ (Supporting Information, Figure S9b, c), the spectra revealed features corresponding to these materials, however, with much diminished peak intensities compared to that shown in the Supoprting Information, Figure S9 at $\lambda_{\text {exc }} 425$ $\mathrm{nm}$. These observations suggest that at $\lambda_{\text {exc }} 370 \mathrm{~nm}$, in addition to CNDs, both $\mathrm{MoS}_{2}$ and $\mathrm{WS}_{2}$ also get excited to some extent. Interestingly, when CND-MoS 2 and CND-WS 2 were excited at $370 \mathrm{~nm}$, the peaks corresponding to the excited CNDs revealed rapid deactivation with simultaneous development of strong excitonic peaks of $\mathrm{MoS}_{2}$ and $\mathrm{WS}_{2}$, more so for CND-MoS $\mathrm{M}_{2}$ than that for CND-WS $\mathrm{W}_{2}$ (Figure 4). These results indicate occurrence of energy transfer from singlet excited CNDs to $\mathrm{MoS}_{2}$ and $\mathrm{WS}_{2}$ in the hybrids. In both hybrids the excitation transfer was complete within 4-5 ps, indicating an efficient process.
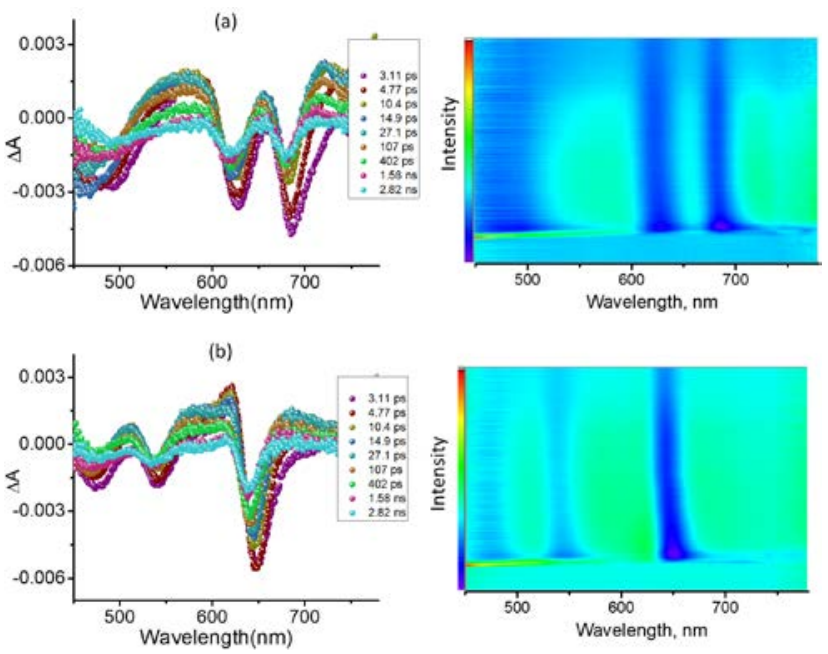

Figure 4. Femtosecond transient spectra of (a) CND-MoS2, and (b) $\mathrm{CND}-\mathrm{WS}_{2}$ in DMF ( $\left.\lambda_{\text {exc }} 370 \mathrm{~nm}\right)$. The right-hand panel shows intensity-wavelength maps.

Next, the hybrids were excited at $425 \mathrm{~nm}$, where majority of $\mathrm{MoS}_{2}$ and $\mathrm{WS}_{2}$ have absorbance. The transient spectral features of CND-MoS 2 (Figure 5a) were distinctly different from that of exfoliated $\mathrm{MoS}_{2}$ (Supporting Information, Figure S8a), especially with respect to the peak time profiles (see intensitywavelength maps). Figure 5 a(iii) shows the time profile of the $688 \mathrm{~nm}$ peak of exfoliated $\mathrm{MoS}_{2}$ and CND-MoS 2 corresponding to the excitonic peak at $688 \mathrm{~nm}$. The recovery of the exciton peak was slow for CND-MoS 2 suggesting occurrence of excited state events from the excited $\mathrm{MoS}_{2}$ to the covalently linked CNDs. Earlier, a facile oxidation was observed for $\operatorname{MoS}_{2}\left(E_{\mathrm{pa}}=\right.$ $-0.08 \mathrm{~V}$ ) suggesting that it could act as an electron donor generating charge separated state. ${ }^{53-56}$ In such an event, charge transfer from a dissociated excitonic state would be promoted to CNDs conduction band, generating a charge separated state. The hole in $\mathrm{MoS}_{2}$ layer would recover slowly due to a charge recombination process. 

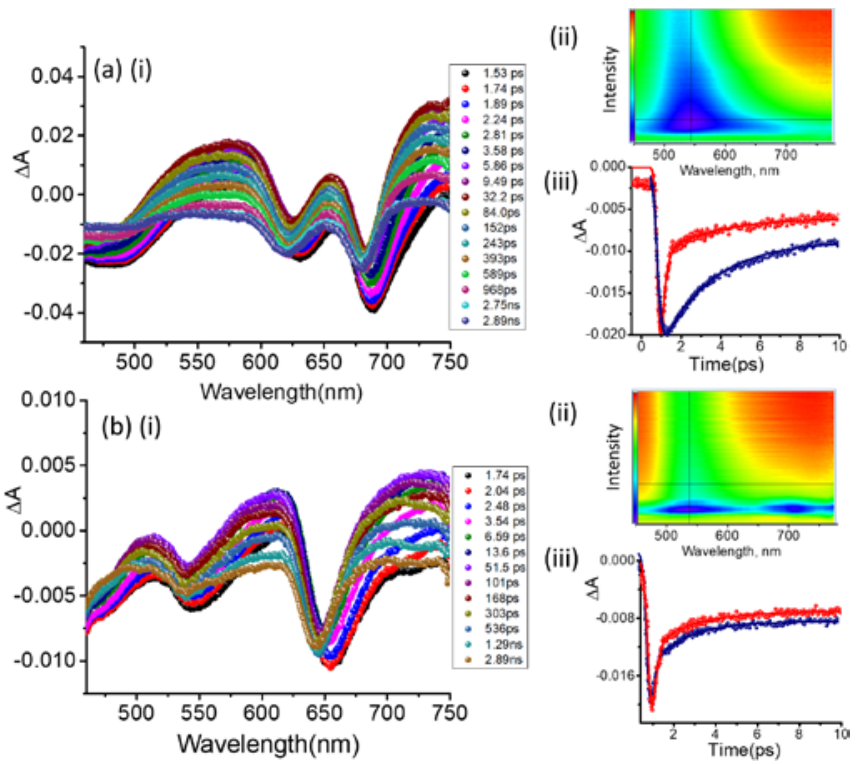

Figure 5. Femtosecond transient spectra of (a) CND-MoS 2 , and (b) $\mathrm{CND}-\mathrm{WS}_{2}$, in DMF ( $\lambda_{\text {exc }} 425 \mathrm{~nm}$ ). The right-hand panels show (ii) intensity-wavelength map and (iii) an overlap time profile of the $688 \mathrm{~nm}$ of CND-MoS 2 (blue) and exfoliated $\mathrm{MoS}_{2}$ (red) and 652 nm of CND-WS 2 (blue) and exfoliated $\mathrm{WS}_{2}$ (red).

Further, the transient data of exfoliated $\mathrm{MoS}_{2}$ and CND-MoS were subjected to global analysis for kinetic evaluations (Figure 6). Decay associated spectra of exfoliated $\mathrm{MoS}_{2}$ revealed three major components; the spectrum at 2.3 ps consists of positive peaks at the spectral range, where excitonic peaks are expected. The spectrum at $1.1 \mathrm{~ns}$ had decay of positive transients. The final component with over 3 ns related to only the excitonic signals with positive shift relative to A exciton. Earlier, for the ultimate decay of the excitons, a lifetime of around 30 ns was established. ${ }^{50}$ Interestingly, for $\mathrm{CND}-\mathrm{MoS}_{2}$, at least 5 components were needed for satisfactory fit. The component at 1 ps was too fast to be assigned to any excited state process as several ultrafast processes such as vibrational cooling, solvent relaxation, etc. occur. The 4.2 and 1.3 ps components had features of excitons in the growth and decay, respectively. The 246 ps component had features in the 450-550 nm range, ascribed to trionic state that revealed faster decay. The longlived decay component had only the excitonic features similar to that observed for unmodified $\mathrm{MoS}_{2}$. These results suggest that the charge separation occurs with a time constant of about 250 ps in the CND-MoS 2 hybrid.
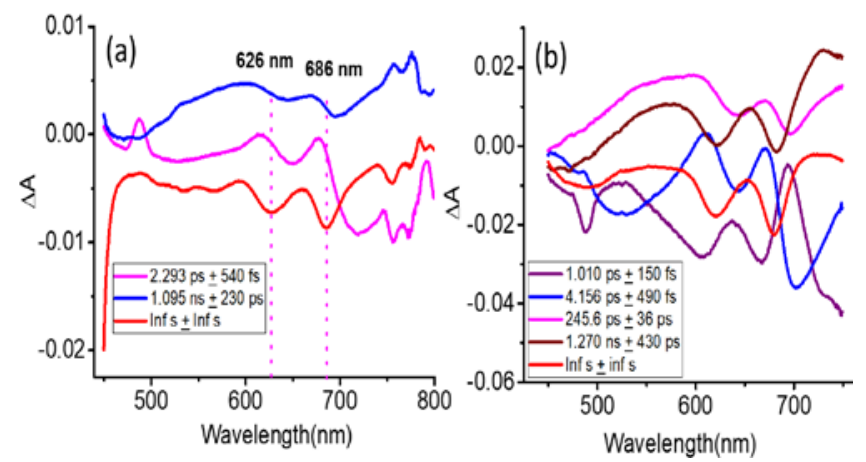

Figure 6. Decay associated spectra of (a) exfoliated $\mathrm{MoS}_{2}$, and (b) CND-MoS 2 for the transient data shown in Figure S7a and Figure 5 a.
Transient spectra recorded for CND-WS 2 (Figure 5b) were close to that of unmodified $\mathrm{WS}_{2}$ (Supporting Information, Figure S8b). The time profiles of the $652 \mathrm{~nm}$ peak (Figure $5 \mathrm{~b}(\mathrm{iii})$ ) were superimposable suggesting lack of major excited state events such as charge transfer from excited $\mathrm{WS}_{2}$ to CNDs. This could be rationalized to the harder oxidation of $\mathrm{WS}_{2}\left(E_{\mathrm{pa}}=\right.$ 0.23 ) that would dampen any charge transfer events due to energy considerations. Hence, no further analysis was performed on this hybrid material.

\section{CONCLUSIONS}

In summary, following the 1,2-dithiolane functionalization protocol for TMDs, the covalent grafting of CNDs onto exfoliated $\mathrm{MoS}_{2}$ and $\mathrm{WS}_{2}$ was accomplished. The newly prepared CND-MoS $\mathrm{C}_{2}$ and $\mathrm{CND}^{-\mathrm{WS}_{2}}$ hybrid materials were fully characterized by complementary spectroscopic, thermal and electron microscopy imaging techniques. Furthermore, electrochemical assays revealed that $\mathrm{MoS}_{2}$ was a better electron donor compared to $\mathrm{WS}_{2}$. Photochemical events upon preferential photoexcitation of CNDs and TMDs were probed. Excitation of CND-MoS ${ }_{2}$ and CND-WS $\mathrm{W}_{2}$ at $370 \mathrm{~nm}$ revealed ultrafast energy transfer from excited CNDs to $\mathrm{MoS}_{2}$ and $\mathrm{WS}_{2}$, while exciting the TMDs within the hybrid materials at $425 \mathrm{~nm}$, charge transfer in $\mathrm{CND}-\mathrm{MoS}_{2}$ but not in $\mathrm{CND}-\mathrm{WS}_{2}$ was seen. The observed excited state electron transfer processes bring us one-step closer to utilize the covalently modified TMDs in donor-acceptor type hybrids for energy harvesting applications.

\section{EXPERIMENTAL SECTION}

Electron microscopy imaging. STEM imaging and EELS studies were conducted using an aberration-corrected FEI Titan Low-Base microscope operated at $80 \mathrm{kV}$. This microscope was equipped with a Cs probe corrector and a Gatan Tridiem ESR 865 electron energy loss spectroscopy (EELS) spectrometer. The energy resolution was $\sim 1 \mathrm{eV}$. The convergence and collection angles were 25 and $50 \mathrm{mrad}$, respectively. The EELS studies were conducted in STEM mode, using the spectrum-line scan mode. To increase the signal/noise ratio of the EEL spectra, the datasets were then de-noised with the open-source program Hyperspy by using principal component analysis routines.

Femtosecond transient absorption spectroscopy. Experiments were performed using an Ultrafast Femtosecond Laser Source (Libra series) by Coherent Inc. (Santa Clara, CA) incorporating diode-pumped, mode locked Ti:Sapphire laser (Vitesse) and diode-pumped intra cavity doubled Nd:YLF laser (Evolution) to generate a compressed laser output of $1.45 \mathrm{~W}$. For optical detection, a Helios transient absorption spectrometer from Ultrafast Systems (Sarasota, FL) was used. The source for the pump and probe pulses were derived from the fundamental output of Libra (Compressed output $1.45 \mathrm{~W}$, pulse width 100 fs) at a repetition rate of $1 \mathrm{kHz}$. About $95 \%$ of the fundamental output of the laser was introduced into a TOPAS-Prime-OPA system with 290-2600 $\mathrm{nm}$ tuning range from Altos Photonics Inc., (Bozeman, MT), while the rest of the output was used for generation of white light continuum. Kinetic traces at appropriate wavelengths were assembled from the time-resolved spectral data. Data analysis was performed using Surface Xplorer software supplied by Ultrafast Systems. All measurements were conducted in degassed solutions at room temperature.

Modified CNDs with lipoic acid. Initially, $2 \mathrm{~g}$ of citric acid (1 equiv.) were dissolved in DMF $(10 \mathrm{~mL})$ and the solution was 
cooled in ice bath. Then, $4.4 \mathrm{~mL}$ of $N, N^{\prime}$-diisopropylcarbodiimide (DIC) as coupling agent (3 equiv.) were added slowly to the solution while stirring, forming a white dispersion. After one minute, $1.9 \mathrm{~mL}$ of ethylenediamine (EDA) in cold water $(10 \mathrm{~mL})$ were poured into the reaction mixture. When the color turned yellow and then orange the fluorescent particles were formed, thus butylamine $(5 \mathrm{~mL})$ was added and the reaction mixture was left under stirring for 24 hours. The addition of butylamine allowed the in-situ introduction of butyl groups on the CNDs surface, enhancing lipophilicity and at the same time consuming all remaining carboxylic groups, thus stopping the growth of the nanoparticles. Then, the solution was filtered to remove the DIC urea byproduct, the filtrate was washed three times with diethylether and the excess of butylamine was removed under vacuum evaporation. Finally, the water phase was purified by dialysis in ultrapure water (molecular weight cut-off $=0.5-1 \mathrm{kDa}, 3$ days). The dry product of CNDs- $\mathrm{NH}_{2}(0.7 \mathrm{~g})$ was obtained as a yellow powder by freeze-drying.

In the next step, CNDs- $\mathrm{NH}_{2}$ (200 mg) were dissolved in a mixture of methanol $(5 \mathrm{~mL})$ and dichloromethane $(25 \mathrm{~mL})$ and cooled to $0{ }^{\circ} \mathrm{C}$. In another flask, lipoic acid (300 mg) was dissolved in dichloromethane $(5 \mathrm{~mL})$, cooled at $0{ }^{\circ} \mathrm{C}$ and 336 mg of (3-dimethylaminopropyl)-N’-ethylcarbodiimide (EDC) were added. After 20 minutes, the lipoic acid solution was poured into that of CNDs- $\mathrm{NH}_{2}$. The reaction mixture was left under stirring for 24 hours and after that period additional lipoic acid (100 mg) and EDC (56 mg) were added. After 3 hours the reaction mixture was extracted with distilled water, aqueous $\mathrm{NaOH}(\mathrm{pH}=11)$ and with brine. The organic phase was dried over $\mathrm{MgSO}_{4}$, the dichloromethane was partially evaporated by vacuum and diluted with ethyl acetate, resulting on the precipitation of the particles (centrifugation at $3200 \mathrm{rpm}, 5$ minutes). The redispersion-precipitation process was repeated until the lipoic acid spot in TLC disappeared. The precipitate was dissolved again in methanol/dichloromethane $1: 1 \mathrm{v} / \mathrm{v}$ and dried over $\mathrm{MgSO}_{4}$ to obtain CNDs carrying 1,2-dithiolane chains as a brown solid, after vacuum evaporation of the solvent.

Exfoliation of semiconducting TMDs. Bulk TMDs (150$200 \mathrm{mg}$ ) were dispersed in chlorosulfonic acid and sonicated for 2 hours at room temperature. The solution was left under stirring during a month, occasionally sonicated for 30 seconds. Afterwards it was added cold water to the solution under stirring, drop by drop and extremely carefully. Please notice that the reaction is exothermic and releases gaseous $\mathrm{HCl}$. Next, the mixture was filtrated on a PTFE filter of $0.2 \mu \mathrm{m}$ pore-size and washed with a good amount of methanol and acetone. The solid compound was added to $\mathrm{N}$-methyl pyrrolidone and sonicated for 1 hour (tip sonication at 30-35\% of amplitude (100\% of 200 W)). After 3 days the supernatant was taken, filtrated on PTFE filter $(0.2 \mu \mathrm{m}$ pore-size $)$ and washed with a large amount of methanol, acetone and dichloromethane.

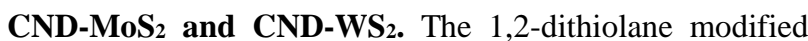
CNDs (50 mg) were dissolved in methanol $(1 \mathrm{~mL})$. In another flask, exfoliated TMDs (20 mg) were dispersed in DMF (10 $\mathrm{mL}$ ) by sonication (10 $\mathrm{min}$ ) and dropped in the CNDs solution. The flask was covered with aluminum foil and the reaction mixture was stirred at $70{ }^{\circ} \mathrm{C}$ for 4 days. After that period, it was cooled and filtered through a PTFE membrane $(0.2 \mathrm{~nm}$ pore size). The solid residue was extensively washed with methanol and dichloromethane to obtain the CND-TMDs.

\section{ASSOCIATED CONTENT}

\section{Supporting Information}

General instrumentation, ${ }^{1} \mathrm{H}$ NMR, ATR-IR, Raman, UV-Vis and femtosecond transient absorption spectra and cyclic voltamograms. The Supporting Information is available free of charge on the ACS Publications website.

\section{AUTHOR INFORMATION}

\section{Corresponding Author}

* Email: tagmatar@eie.gr (N. Tagmatarchis)

* Email: Francis.DSouza@unt.edu (F. D’Souza)

* Email: wmaser@icb.csic.es (W. K. Maser)

*Email: arenal@unizar.es (R. Arenal)

\section{Author Contributions}

\# These authors contributed equally.

\section{ACKNOWLEDGMENT}

This project has received funding from the European Union's Horizon 2020 research and innovation programme under the Marie Sklodowska-Curie grant agreement $\mathrm{N}^{\circ}$ 642742. Support of this work by the project "Advanced Materials and Devices" (MIS 5002409) which is implemented under the "Action for the Strategic Development on the Research and Technological Sector", funded by the Operational Programme "Competitiveness, Entrepreneurship and Innovation" co-financed by Greece (Ministry of Economy and Development, NSRF 2014-2020) and EU (European Regional Development Fund) to N. T. and the US-NSF (grant 1401188 to F. D.) is acknowledged. The HR-STEM and STEM-EELS studies were conducted at the Laboratorio de Microscopias Avanzadas, Universidad de Zaragoza, Spain. R. A. acknowledges support from Spanish MINECO grant MAT201679776-P (AEI/FEDER, UE) and from EU H2020 "Graphene Flagship” grant agreement 785219. W. K. M. and A. M. B. acknowledge Spanish MINECO grant ENE2016-79282-C5-1-R (AEI/FEDER, EU) and the Covernment of Aragon through project DGA-T03_17R (FEDER, EU).

\section{ABBREVIATIONS}

CNDs, carbon nanodots; exTTF, extended tetrathiafulvalene; TMDs, transition metal dichalcogenides; TGA, thermogravimetric analysis; TEM, transmission electron microscopy; EELS, electron energy loss spectroscopy; HAADF, high angle annular dark field; STEM, scanning transmission electron microscopy; EDS, energy dispersive X-ray spectroscopy.

\section{REFERENCES}

(1) Margraf, F. J.; Lodermeyer, T.; Strauss, V.; Haines, P.; Walter, J.; Peukert, W.; Costa, R. D.; Clark, T.; Guldi, D. M. Using carbon nanodots as inexpensive and environmentally friendly sensitizers in mesoscopic solar cells. Nanoscale Horiz. 2016, 1, 220-226.

(2) Martindale, B. C. M.; Joliat, E.; Bachmann, C.; Alberto, R.; Reisner, E. Clean donor oxidation enhances rhe $\mathrm{H}_{2}$ evolution activity of a carbon quantum dot-molecular catalyst photosystem. Angew. Chem. Int. Ed. 2016, 55, 9402-9406.

(3) Li, X.; Rui, M.; Song, J.; Shen, Z.; Zeng, H. Carbon and graphene quantum dots for optoelectronic and energy devices: A review. Adv. Funct. Mater. 2015, 25, 4929-4947.

(4) Data, K. K. R.; Qi, G.; Zboril, R.; Giannelis, E. P. Yellow emitting carbon dots with superior colloidal, thermal, and photchemkical stabilities. J. Mater. Chem. C 2016, 4, 97989803. 
(5) Gu, J.; Hu, D.; Huang, J.; Zhang, Q.; Jia, X.; Xi, K. One-pot synthesis and control of aqueous soluble and organic soluble carbon dots from a designable waterborne polyurethane emulsion. Nanoscale 2016, 8, 3973-3981.

(6) Zhang, J.; Yu, S. -H. Carbon dots: Large-scale synthesis, sensing and bioimaging. Mater. Today 2016, 19, 382-393.

(7) Cayuela, A.; Soriano, M. L.; Carillo-Crion, C.; Valcarcel, M. Semiconductor and carbon-based fluorescent nanodots: The need for consistency. Chem. Commun. 2016, 52, 1311-1326.

(8) Das, R.; Bandyopadhyay, R.; Pramanik, P. Carbon quantum dots from natural resource: A review. Mater. Today Chem. 2018, 8, 96-109

(9) Zheng, X. T.; Ananthanarayanan, A.; Luo, K. Q.; Chen, P. Glowing graphene quantum dots and carbon dots: Properties, Syntheses, and biological applications. Small 2015, 11, 16201636.

(10) Zhai, X.; Zhang, P.; Liu, C.; Bai, T.; Li, W.; Dai, L.; Liu, W. Highly luminescent carbon nanodots by microwave-assisted pyrolysis. Chem. Commun. 2012, 48, 7955-795.

(11) Skaltsas, T.; Stergiou, A.; Chronopoulos, D. D.; Zhao, S.; Shinohara, H.; Tagmatarchis, N. All-carbon nanosized hybrid materials: Fluorescent carbon dots conjugated to multiwalled carbon nanotubes. J. Phys. Chem. C 2016, 120, 8550-8558.

(12) Arcudi, F.; Strauss, V.; Dordevic, L.; Cadranel, A.; Guldi, D. M.; Prato, M. Porphyrin antennas on carbon nanodots: Excited state energy and electron transduction. Angew. Chem. Int. Ed. 2017, 56, 12097-12101.

(13) Ferrer-Ruiz, A.; Schari, T.; Haines, P.; Rodriguez-Perez, L.; Cadranel, A.; Herranz, M. A.; Guldi, D. M.; Martin, N. Exploring tetrathiafulvalene-carbon nanodot conjugates in charge transfer reactions. Angew. Chem. Int. Ed. 2018, 57, 1001-1005.

(14) Baker, S. N.; Baker, G. A. Luminescent carbon nanodots: Emerging nanolights. Angew. Chem. Int. Ed. 2010, 49, 67266744.

(15) Strauss, V.; Margraf, J. T.; Dolle, C.; Butz, B.; Nacken, T. J.; Walter, J.; Bauer, W.; Peukert, W.; Spiecker, E.; Clark, T.; Guldi, D. M. Carbon nanodots: Toward a comprehensive understanding of their photoluminescence. J. Am. Chem. Soc. 2014, 136, 17308-17316.

(16) Strauss, V.; Margraf, J. T.; Clark, T.; Guldi, D. M. A carboncarbon hybrid - immobilizing carbon nanodots onto carbon nanotubes. Chem. Sci. 2015, 6, 6878-6885.

(17) Strauss, V.; Margraf, J. T.; Dirian, K.; Syrgiannis, Z.; Prato, M.; Wessendorf, C.; Hirsch, A.; Clark, T.; Guldi, D. M. Carbon nanodots: Supramolecular electron donor-acceptor hybrids featuring perylenediimides. Angew. Chem. Int. Ed. 2015, 54, 8292-8297.

(18) Cadranel, A.; Strauss, V.; Margraf, J. T.; Winterfeld, K. A.; Vogl, C.; Dordevic, L.; Arcudi, F.; Hoelzel, H.; Jux, N.; Prato, M.; Guldi, D. M. Screening supramolecular interactions between carbon nanodots and porphyrins. J. Am. Chem. Soc. 2018, 140, 904-907.

(19) Pumera, M.; Sofer, Z.; Ambrosi, A. Layered transition metal dichalcogenides for electrochemical energy generation and storage. J. Mater. Chem. A 2014, 2, 8981-8987.

(20) Perivoliotis, D. K.; Tagmatarchis, N. Recent advancements in metal-based hybrid electrocatalysts supported on graphene and related 2D materials for the oxygen reduction reaction. Carbon 2017, 118, 493-510.

(21) Lv, R.; Robinson, J. A.; Schaak, R. E.; Sun, D.; Sun, Y.; Mallouk, T. E.; Terrones, M. Transition metal dichalcogenides and beyond: Synthesis, properties, and applications of singleand few-layer nanosheets. Acc. Chem. Res. 2015, 48, 56-64.

(22) Jariwala, D.; Sangwan, V. K.; Lauhon, L. J.; Marks, T. J.; Hersam, M. C. Emerging applications for semiconducting two-dimensional transition metal dichalcogenides. ACS Nano 2014, 8, 1102-1120.
(23) Wang, Q. H.; Kalantar-Zadeh, K.; Kis, A.; Coleman, J. N.; Strano, M. S. Electronics and optoelectronics of two-dimensional transition metal dichalcogenides. Nat. Nanotechnol. 2012, 7, 699

(24) Duong, D. L.; Yun, S. J.; Lee, Y. H. van der Waals layered materials: Opportunities and challenges. ACS Nano 2017, 11, 11803-11830.

(25) Miro, P.; Audiffred, M.; Heine, T. An atlas of two-dimensional materials. Chem. Soc. Rev. 2014, 43, 6537-6554.

(26) Chhowalla, M.; Shin, H. S.; Eda, G.; Li, L. J.; Loh, K. P.; Zhang, $\mathrm{H}$. The chemistry of two-dimensional layered transition metal dichalcogenide nanosheets. Nat. Chem. 2013, 5, 263275.

(27) Grayfer, E. D.; Kozlova, M. N.; Fedorov, V. E. Colloidal 2D nanosheets of $\mathrm{MoS}_{2}$ and other transition metal dichalcogenides through liquid-phase exfoliation. Adv. Colloid Interface Sci. 2017, 245, 40-61.

(28) Tao, H.; Zhang, Y.; Gao, Y.; Sun, Z.; Yan, C.; Texter, J. Scalable exfoliation and dispersion of two-dimensional materials an update. Phys. Chem. Chem. Phys. 2017, 19, 921-960.

(29) Niu, L.; Coleman, J. N.; Zhang, H.; Shin, H.; Chhowalla, M.; Zheng, Z. Production of two-dimensional nanomaterials via liquid-based direct exfoliation. Small 2016, 12, 272-293.

(30) Zhang, X.; Lai, Z.; Tan, C.; Zhang, H. Solution-processed twodimensional MoS2 nanosheets: Preparation, hybridization, and applications. Angew. Chem. Int. Ed. 2016, 55, 8816-8838.

(31) Fan, X.; Xu, P.; Li, Y. C.; Zhou, D.; Sun, Y.; Nguyen, M. A. T.; Terrones, M.; Mallouk, T. E. Controlled exfoliation of $\mathrm{MoS}_{2}$ crystals into trilayer nanosheets. J. Am. Chem. Soc. 2016, 138, 5143-5149.

(32) Pagona, G.; Bittencourt, C.; Arenal, R.; Tagmatarchis, N. Exfoliated semiconducting pure $2 \mathrm{H}-\mathrm{MoS}_{2}$ and $2 \mathrm{H}-\mathrm{WS}_{2}$ assisted by chlorosulfonic acid. Chem. Commun. 2015, 51, 1295012953.

(33) Ryder, C. R.; Wood, J. D.; Wells, S. A.; Hersam, M. C. Cehmically tailoring semiconducting two-dimensional transition metal dichalcogenides and black phosphorus. ACS Nano 2016, 10, 3900-3917.

(34) Bertolazzi, S.; Gobbi, M.; Zhao, Y.; Backes, C.; Samori, P. Molecular chemistry approaches for tuning the properties of two-dimensional transition metal dichalcogenides. Chem. Soc. Rev. 2018, 47, 6845-6888.

(35) Xin, C.; McDonald. A. R. Functionalization of two-dimensional dichalcogenides. Adv. Mater. 2016, 28, 5738-5746.

(36) Knirsch, K. C.; Berner, N. C.; Nerl, H. C.; Cucinotta, C. S.; Gholamvand, Z.; McEvoy, N.; Wang, Z.; Abramovic, I.; Vecera, P.; Halik, M.; Sanvito, S.; Duesberg, G. S.; Nicolosi, V.; Hauke, F.; Hirsch, A.; Coleman, J. N.; Backes, C. Basalplane functionalization of chemically exfoliated molybdenum disulfide by diazonium salts. ACS Nano 2015, 9, 6018-6030.

(37) Backes, C.; Berner, N. C.; Chen, X.; Lafargue, P.; LaPlace, P.; Freeley, M.; Duesberg, G. S.; Coleman, J. N.; McDonald, A. R. Functionalization of liquid-exfoliated two-dimensional $2 \mathrm{H}-$ $\mathrm{MoS}_{2}$. Angew. Chem. Int. Ed. 2015, 54, 2638-2642.

(38) McAdams, S. G.; Lewis, E. A.; Brent, J. R.; Haigh, S. J.; Thomas, A. G.; O'Brien, P.; Tuna, F.; Lewis, D. J. Dual functionalization of liquid-exfoliated semiconducting $2 \mathrm{H}-\mathrm{MoS}_{2}$ with lanthanide complexes bearing magnetic and luminescence properties. Adv. Funct. Mater. 2017, 27, 1703646.

(39) Canton-Vitoria, R.; Sayed-Ahmad-Baraza, Y.; Pelaez-Fernandez, M.; Arenal, R.; Bittencourt, C.; Ewels, C. P.; Tagmatarchis, N. Functionalization of $\mathrm{MoS}_{2}$ with 1,2-dithiolanes: Toward donor-acceptor nanohybrids for energy conversion. NPJ 2D Mater. App. 2017, 1, 13.

(40) Canton-Vitoria, R.; Vallan, L.; Urriolabeitia, E.; Benito, A. M.; Maser, W. K.; Tagmatarchis, N. Electronic interactions in illuminated carbón dot/MoS $\mathrm{S}_{2}$ ensembles and electrocatalytic activity towards hydrogen evolution. Chem. Eur. J. 2018, 24, 10468-10474.

(41) Canton-Vitoria, R.; Stangel, C.; Tagmatarchis, N. Electrostatic association of ammonium-functionalized layered-transition- 
metal dichalcogenides with an anionic porphyrin. ACS Appl. Mater. Interfaces 2018, 10, 23476-23480.

(42) Li, H.; Zhang, Q.; Chong, R. Y. C.; Beng, K. T.; Hang, T. E. T.; Olivier, A.; Baillargeat. D. From bulk to monolayer $\mathrm{MoS}_{2}$ : Evolution of Raman scattering. Adv. Funct. Mater. 2012, 22, 1385-1390.

(43) Benson, E. E.; Zhang, H.; Schuman, S. A.; Nanayakkara, S. U.; Bronstein, N. D.; Ferrere, S.; Blackburn, J. L.; Miller, E. M. Balancing the hydrogen evolution reaction, surface energietics, and stability of metallic $\mathrm{MoS}_{2}$ nanosheets via covalent functionalization. J. Am. Chem. Soc. 2018, 140, 441-450.

(44) Nayak, A. P.; Pandey, T.; Voiry, D.; Liu, J.; Moran, S. T.; Sharma, A.; Tan, C.; Chen, C.-H.; Li, L.-J.; Chhowalla, M.; Lin, J.-F.; Singh, A. K.; Akinwande, D. Pressure-dependent optical and vibrational properties of monolayer molybdenum disulfide. Nano Lett. 2015, 15, 346-353.

(45) Jimenez Sandoval, S.; Yang, D.; Frindt, R. F.; Irwin, J. C. Raman study and lattice dynamics of single molecular layers of $\mathrm{MoS}_{2}$. Phys. Rev. B 1991, 44, 3955-3962.

(46) Chakraborty, B.; Bera, A.; Muthu, D. V. S.; Bhowmick, S.; Waghmare, U. V.; Sood, A. K. Symmetry-dependent phonon renormalization in monolayer $\mathrm{MoS}_{2}$. Phys. Rev. B: Condens. Matter. 2012, 85, 161403.

(47) Shi, Y.; Huang, J.-K.; Jin, L.; Hsu, Y.-T.; Yu, S. F.; Li, L.-J.; Yang, H. Y. Selective decoration of Au nanoparticles on monolayer $\mathrm{MoS}_{2}$ single crystals. Sci. Rep. 2013, 3, 1839.

(48) Arenal, R.; De Matteis, L.; Custardoy, L.; Mayoral, A.; Tence, M.; Grazu, V.; de la Fuente, J. M.; Marquina, C.; Ibarra, M. R. Spatially-resolved EELS analysis of antibody distribution on biofunctionalzied magnetic nanoparticles. ACS Nano 2013, 7, 4006-4013.

(49) Arenal, R.; March, K.; Ewels, C. P.; Rocquefelte, X.; Kociak, M.; Loiseau, A.; Stephan, O. Atomic configuration of nitrogendoped single-walled carbon nanotubes. Nano Lett. 2014, 14, 5509-5516.
(50) Wibmer, L.; Lages, S.; Unruh, T.; Guldi, D. M. Excitons and trions in one-photon- and two-photon-excited $\mathrm{MoS}_{2}$ : A study in dispersions. Adv. Mater. 2018, 30, 1706702.

(51) Chowdhury, R. K.; Nandy, S.; Bhattacharya, S.; Karmakar, M.; Bhaktha, B. N. S.; Datta, P. K.; Taraphder, A.; Ray, S. K. Broadband pump-probe study of biexcitons in chemically exfoliated layered $\mathrm{WS}_{2}$. Cond. Mater. 2018, 1-8.

(52) Jiang, T.; Chen, R.; Zheng, X.; Xu, Z.; Tang, Y. Photo-induced excitonic structure renormalization and broadband absorption in monolayer tungsten disulphide. Optics Expr. 2018, 26, 859869.

(53) Homan, S. B.; Sangwan, V. K.; Balla, I.; Bergeron, H.; Weiss, E. A.; Hersam, M. C. Ultrafast exciton dissociation and longlived charge separation in a photovoltaic pentacence- $\mathrm{MoS}_{2}$ van der Waals heterojunction. Nano Lett. 2017, 17, 164-169.

(54) Kafle, T. R.; Kattel, S.; Lane, S. D.; Wang, T.; Zhao, H.; Chan, W.-L. Charge-transfer exciton and spin flipping at organictransition-metal dichalcogenide interfaces. ACS Nano 2017, 11, 10184-10192.

(55) Nguyen, E. P.; Carey, B. J.; Harrison, C. J.; Atkin, P.; Berean, K. J.; Gaspera, E. D.; Ou, J. Z.; Kaner, R. B.; Kalantar-zadeh, K.; Daeneke, T. Excitation dependent bidirectional electron transfer in phthalocyanine $\mathrm{MoS}_{2}$ nanosheets. Nanoscale 2016, 8, 16276-16283.

(56) Choi, J.; Zhang, H.; Choi, J. H. Modulating optoelectronic properties of two-dimensional transition metal dichalcogenide semiconductors by photoinduced charge transfer. ACS Nano 2016, 10, 1671-1680. 
ToC

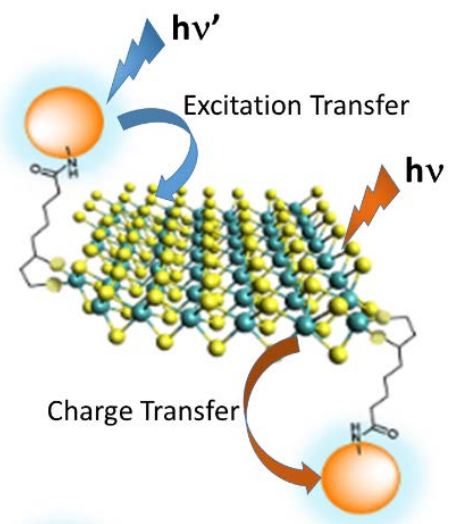

